\title{
Colour stabilities of three types of orthodontic clear aligners exposed to staining agents
}

\author{
Chen-Lu Liu, Wen-Tian Sun, Wen Liao, Wen-Xin Lu, Qi-Wen Li, Yunho Jeong, Jun Liu and Zhi-He Zhao
}

The aim of this study was to evaluate and compare the colour stabilities of three types of orthodontic clear aligners exposed to staining agents in vitro. Sixty clear orthodontic aligners produced by three manufacturers (Invisalign, Angelalign, and Smartee) were immersed in three staining solutions (coffee, black tea, and red wine) and one control solution (distilled water). After 12-h and 7-day immersions, the aligners were washed in an ultrasonic cleaner and measured with a colourimeter. The colour changes $\left(\Delta E^{*}\right)$ were calculated on the basis of the Commission Internationale de l'Eclairage $L^{*} a^{*} b^{*}$ colour system (CIE $\left.L^{*} a^{*} b^{*}\right)$, and the results were then converted into National Bureau of Standards (NBS) units. Fourier transformation infrared (FT-IR) spectroscopy and scanning electron microscopy (SEM) were conducted to observe the molecular and morphologic alterations to the aligner surfaces, respectively. The three types of aligners exhibited slight colour changes after $12 \mathrm{~h}$ of staining, with the exception of the Invisalign aligners stained with coffee. The Invisalign aligners exhibited significantly higher $\Delta E^{*}$ values (ranging from 0.30 to 27.81 ) than those of the Angelalign and Smartee aligners $\left(\Delta E^{*}\right.$ values ranging from 0.33 to 1.89 and 0.32 to 1.61 , respectively, $\boldsymbol{P}<0.05)$. FT-IR analysis confirmed that the polymer-based structure of aligners did not exhibit significant chemical differences before and after the immersions. The SEM results revealed different surface alterations to the three types of aligner materials after the 7-day staining. The three types of aesthetic orthodontic appliances exhibited colour stability after the 12-h immersion, with the exception of the Invisalign aligners stained by coffee. The Invisalign aligners were more prone than the Angelalign and Smartee aligners to pigmentation. Aligner materials may be improved by considering aesthetic colour stability properties. International Journal of Oral Science (2016) 8, 246-253; doi:10.1038/ijos.2016.25; published online 23 September 2016

Keywords: aesthetics; clear aligner; clear aligner material; colour stability; orthodontic appliance

\section{INTRODUCTION}

The aesthetic considerations associated with social perceptions influence orthodontic treatment. ${ }^{1-2}$ The increasing demand for more aesthetic orthodontic appliances has elicited an aesthetic revolution marked by the emergence of invisible appliances, such as aesthetic brackets, lingual appliances, and clear aligners. ${ }^{3-5}$ Among these appliances, clear aligners are often preferred over brackets by adults, owing to aesthetic and comfort considerations. ${ }^{6-7}$

The clear aligner treatment process is based on the sequential use of aligners made of transparent thermoplastic materials. ${ }^{8}$ Various thermoplastic materials are currently used for fabrication, ${ }^{9}$ including polyvinyl chloride, ${ }^{10}$ polyurethane (PU), ${ }^{11}$ polyethylene terephthalate (PET), ${ }^{12}$ and polyethylene terephthalate glycol (PETG). ${ }^{10,12}$ Many studies have been conducted on clear aligners, particularly focusing on the mechanical properties of the aligners ${ }^{13-15}$ or the biomechanics of tooth movement during orthodontic treatment. ${ }^{12,16}$ Researchers have also investigated new clear aligner materials to obtain desirable mechanical properties for orthodontic treatment. ${ }^{17}$ However, although clear aligners are promising aesthetic orthodontic appliances, the aesthetic stability of clear aligner materials has scarcely been reported.

From an aesthetic perspective, the colour stability and transparency of orthodontic clear aligners should be stable during the 2-week orthodontic treatment periods. ${ }^{18-19}$ However, the colour stability of dental materials is often influenced by various factors, such as ultraviolet irradiation, staining beverages, and mouthwashes. ${ }^{20}$ Studies have reported that PU-based elastomeric ligatures are vulnerable to pigment adsorption from food and drink in the oral cavity. ${ }^{21-23}$ During usage, it is recommended that aligners be removed before eating and drinking. However, studies have reported that patient compliance regarding removable orthodontic appliances is insufficient, ${ }^{24}$ and this often is a matter of concern for orthodontists. The exposure of the aligner to staining agents in the oral cavity is inevitable, especially when users drink without taking the aligners out of their mouths because of time constraints during work. Statistics

State Key Laboratory of Oral Diseases, Department of Orthodontics, West China Hospital of Stomatology, Sichuan University, Chengdu, China

Correspondence: Professor ZH Zhao, State Key Laboratory of Oral Diseases, Department of Orthodontics, West China Hospital of Stomatology, Sichuan University, No.14, Section 3, Renmin South Road, Chengdu 610041, China

E-mail: zhzhao@scu.edu.cn

Dr J Liu, State Key Laboratory of Oral Diseases, Department of Orthodontics, West China Hospital of Stomatology, Sichuan University, No.14, Section 3, Renmin South Road, Chengdu 610041, China

E-mail: junliu@scu.edu.cn

Accepted 3 May 2016 
have demonstrated that over 50\% of American adults drink coffee every day and that their average consumption is 3.2 cups per day. ${ }^{25}$ For patients who do not follow the instructions, the pigments in staining agents might accumulate and lead to colour changes in the aligner materials. Thus, clear aligners might become less aesthetically appealing even during the 2 -week treatments, ${ }^{26}$ and this is a clinical concern. Therefore, there is a need to investigate the colour stabilities of commonly used types of aligners to provide evidence for both patients and orthodontists regarding clinical aesthetic considerations and instructions.

The aim of the present study was to evaluate and compare the colour stabilities of three types of orthodontic clear aligners before and after exposure to staining solutions in vitro.

\section{MATERIALS AND METHODS}

\section{Staining of the aligners}

Three sets of polyvinyl siloxane impressions of the upper dentition were taken from a subject. The aligners (and/or retainers) made by thermoplastic materials were manufactured by three companies (Invisalign, Align Technology, Santa Clara, CA, USA; Angelalign, EA Medical Instruments, Shanghai, China; and Smartee, Smartee Denti-Technology, Shanghai, China). According to previous studies and the material safety data sheets provided by the manufacturers, the Invisalign aligner is made of SmartTrack material, which is primarily composed of thermoplastic PU with some modification (the details are not disclosed by the manufacturer). ${ }^{11,27-29}$ The Smartee aligner is manufactured with Erkodur foil (Erkodent Erich Kopp GmbH, Pfalzgrafenweiler, Germany), which is composed of PETG. ${ }^{30}$

Because coffee, black tea, and red wine have been reported to cause more severe stains than other beverages, ${ }^{31}$ the present study used these liquids as staining agents and distilled water as a control. ${ }^{32}$ The coffee (G7 Pure Black Instant Coffee; Trung Nguyen, Bac giang, Vietnam) and tea (Yellow Label Tea; Lipton, Hefei, China) solutions were prepared according to the methods used in previous studies. ${ }^{23}$ The concentrations were $3 \mathrm{~g}$ of coffee powder or one teabag per $100 \mathrm{~mL}$ boiling distilled water. ${ }^{23}$ Undiluted red wine (Cabernet Sauvignon red wine; Saflam, Yantai, China) was used. Twenty aligners (and/or retainers) from each manufacturer were randomly divided into four groups according to the solutions. The aligners were immersed in each solution in a water bath at $37^{\circ} \mathrm{C}$ for $12 \mathrm{~h}$ or 7 days. The solutions were refreshed every day.

\section{Colour change evaluations}

The colour changes were characterised according to the Commission Internationale de I'Eclairage $L^{\star} a^{\star} b^{\star}$ colour system (CIE $\left.L^{\star} a^{\star} b^{\star}\right) .^{33}$ The colour parameter $L^{\star}$ represents lightness (+ bright, - dark), $a^{*}$ represents the red $(+)$ to green $(-)$ colour scale, and $b^{*}$ indicates the yellow $(+)$ to blue $(-)$ colour scale. ${ }^{34}$ The colour parameters $L^{*}$, $a^{*}$, and $b^{*}(t=0,1,2)$ of aligners were measured with a standard VITA Easyshade Compact $^{30}$ colourimeter (Vita Zahnfabrik, Bad Sackingen, Germany) before staining $\left(T_{0}\right)$ and after periods of $12 \mathrm{~h}\left(T_{1}\right)$ and 7 days $\left(T_{2}\right)$ of staining. All of the aligners were washed in an ultrasonic cleaner for $5 \mathrm{~min}$ and dried with tissue paper before the measurements. Standard measurements were performed by an investigator who was blind to the group division. The flat labial surface of the right upper central incisor of each aligner was measured. A tooth model made using flowable resin (Filtek Universal Restorative; 3M ESPE, Saint Paul, MN, USA) with an A3 Body Shade as was used as the background reference and set behind the labial surface of each aligner. ${ }^{19}$ The measurements were performed by firmly contacting the optical sensor tip vertically to the flat surface of the aligner. All measurements were conducted in the same room with standardized illumination. The total colour change $\left(\Delta E^{\star}\right)$ value was calculated according to the equation $\Delta E^{*}=\left[\left(\Delta L^{*}\right)^{2}+\left(\Delta a^{*}\right)^{2}+\left(\Delta b^{*}\right)^{2}\right]^{1 / 2}$, which represents the colour difference before and after staining. ${ }^{33} \Delta L^{*}, \Delta a^{*}$ and $\Delta b^{*}(t=1,2)$ are the subtractions of the $L^{*}, a^{*}$ and $b^{*}$ colour parameters, respectively, between $T_{1}-T_{0}$ and $T_{2}-T_{0}$.

\section{Colour change rating}

The national bureau of standards (NBS) system was used to describe levels of perceptible colour change upon visual inspection (Table 1)..$^{23,35}$ The $\Delta E^{*}$ values were converted into NBS units with the equation $\mathrm{NBS}=\Delta E^{*} \times 0.92$ to relate the colour changes to a clinical standard. ${ }^{19,36-37}$

\section{Fourier transformation infrared analysis}

Because coffee caused the most marked colour changes, the molecular changes to the aligners' surfaces following coffee staining were examined with Fourier transformation infrared (FT-IR) spectroscopy. ${ }^{27-28}$ Before and after 7 days of staining in coffee, part of the material $(2 \mathrm{~mm} \times$ $2 \mathrm{~mm})$ was cut from the aligner $(n=3)$. The specimens were washed in ultrasonic cleaners for $5 \mathrm{~min}$ and dried with tissue paper. The spectra of the three types of aligners were recorded with an FT-IR spectrometer (Alpha, BRUKER OPTIC GMBH, Ettlingen, Germany) and averaged over 40 scans between 4000 and $500 \mathrm{~cm}^{-1}$ with a resolution of $4 \mathrm{~cm}^{-1}$. The FT-IR spectra were compared with data in the ATR-FTIR LIBRARY COMPLETE OF BRUKER (BRUKER OPTIC GMBH, Ettlingen, Germany).

\section{Scanning electron microscopy examination}

Because coffee caused the most marked staining, scanning electron microscopy (SEM) examinations were conducted to further investigate the surface alternations to the materials that were caused by coffee staining. Small specimens $(2 \mathrm{~mm} \times 2 \mathrm{~mm})$ were cut from coffee-stained aligners $(n=3)$ and then investigated with SEM (INSPECT F; FEI, Brno, Czech) at $\times 50000$ magnifications.

\section{Statistics analysis}

The $\Delta E^{\star}$ values of each group at $12 \mathrm{~h}$ and 7 days were analysed via two-way analyses of variance (ANOVAs), and one-way ANOVAs. Tukey's multiple comparison tests were conducted to investigate the effects of each solution on the materials' $\Delta E^{\star}$ values.

\section{RESULTS}

\section{Colour change evaluations}

Photographs showing the colour change of the aligners before and after immersion in coffee, black tea, red wine, and distilled water are provided in Figure 1. Visual inspection indicated that all three types of aligners exhibited almost no colour change after the 12-h staining, with the exception of the Invisalign aligners in coffee. As the immersion time increased to 7 days, the aligners exhibited enhanced colour changes. The Invisalign aligners exhibited more marked colour changes

\section{Table 1 National bureau of standards ratings}

\begin{tabular}{ll} 
National bureau of standards units & Descriptions of colour changes \\
\hline $0.0-0.5$ & Trace: extremely slight change \\
$0.5-1.5$ & Slight: slight change \\
$1.5-3.0$ & Noticeable: perceivable \\
$3.0-6.0$ & Appreciable: marked change \\
$6.0-12.0$ & Much: extremely marked change \\
12.0 or more & Very much: change to other colour
\end{tabular}




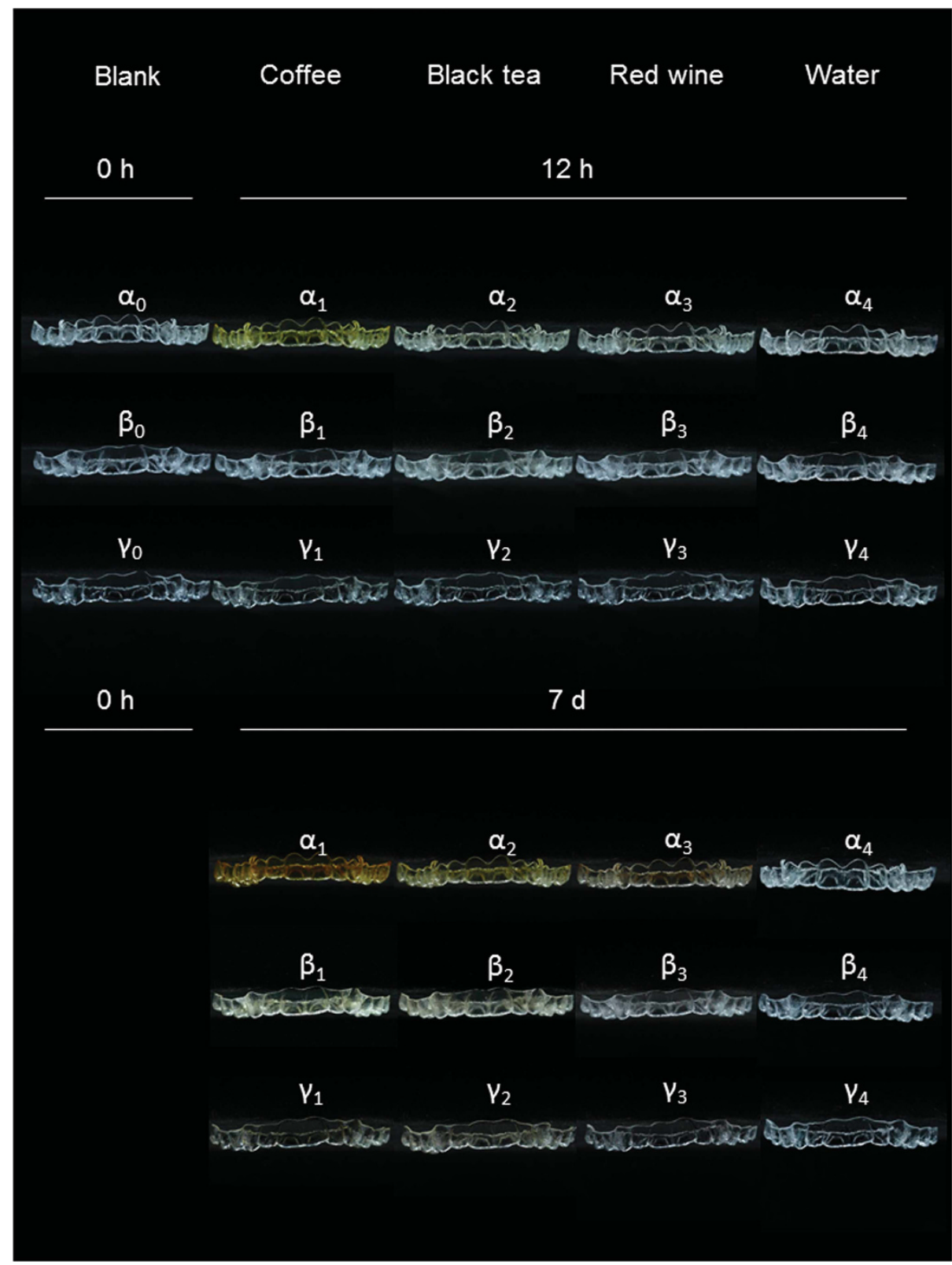

Figure 1 Photographs of the aligners. $\alpha$, Invisalign; $\beta$, Angelalign; $\gamma$, Smartee. Aligners before staining $\left(\alpha_{0}, \beta_{0}\right.$, and $\left.\gamma_{0}\right)$ and after staining in coffee $\left(\alpha_{1}, \beta_{1}\right.$, and $\left.\gamma_{1}\right)$, black tea $\left(\alpha_{2}, \beta_{2}\right.$, and $\left.\gamma_{2}\right)$, red wine $\left(\alpha_{3}, \beta_{3}\right.$, and $\left.\gamma_{3}\right)$, and distilled water $\left(\alpha_{4}, \beta_{4}\right.$, and $\left.\gamma_{4}\right)$ for $12 \mathrm{~h}$ and $7 \mathrm{~d}$.

than the Angelalign and the Smartee aligners in the staining solutions. No perceivable colour changes were detected in any of the three types of aligners after immersion in distilled water even after 7 days.

Quantitative evaluations were further conducted for the colour measurements. The means and standard deviations of the $\Delta E^{*}$ (the colour change) values are presented in Table 2 and Figure 2. Analysis of the $\Delta E^{*}$ values with two-way ANOVA identified significant differences in the colour changes among the different materials $(P<0.05)$ and the different staining solutions $(P<0.05)$, as well as an interaction effect $(P<0.05)$ at each staining time point. The intergroup comparisons of the aligner types in each solution revealed that the Invisalign aligners exhibited significantly higher $\Delta E^{*}$ values than the Angelalign and Smartee aligners $(P<0.05)$, and there was no significant difference between the Angelalign and Smartee aligners (Table 2).

\section{NBS rating}

According to the NBS ratings (Table 1 and Figure 3), after $12 \mathrm{~h}$ of staining, all three types of aligners exhibited only extremely slight or slight colour changes. However, the Invisalign aligners stained with coffee exhibited marked colour change. After 7 days of staining, the Invisalign aligners exhibited extremely marked colour changes, particularly the aligners that were immersed in coffee, whereas the Angelalign and Smartee aligners exhibited only slightly perceivable colour changes. All three types of aligners maintained good colour stability in distilled water even after the 7-day immersion.

\section{FT-IR analysis}

FT-IR spectra of the three types of aligners before and after immersion in coffee were analysed, ${ }^{38-40}$ and the IR peaks of the different groups are 
Table 2 Comparisons of colour change $\left(\Delta E^{*}\right)$ values of three types of aligners in each solution at each time point

\begin{tabular}{|c|c|c|c|c|c|c|c|c|c|c|c|c|}
\hline \multirow[b]{2}{*}{ Brands } & \multicolumn{3}{|c|}{ Coffee } & \multicolumn{3}{|c|}{ Black tea } & \multicolumn{3}{|c|}{ Red wine } & \multicolumn{3}{|c|}{ Water } \\
\hline & Mean & SD & $\mathrm{IC}^{*}$ & Mean & SD & $I C^{*}$ & Mean & SD & $I C^{*}$ & Mean & SD & $I C^{*}$ \\
\hline Invisalign & 6.19 & 0.23 & $\mathrm{a}$ & 1.42 & 0.27 & a & 1.24 & 0.15 & a & 0.40 & 0.16 & $a$ \\
\hline Angelalign & 0.69 & 0.20 & $b$ & 0.63 & 0.16 & $\mathrm{~b}$ & 0.66 & 0.15 & $b$ & 0.33 & 0.13 & a \\
\hline Smartee & 0.61 & 0.16 & $\mathrm{~b}$ & 0.46 & 0.12 & $\mathrm{~b}$ & 0.45 & 0.11 & $\mathrm{~b}$ & 0.32 & 0.19 & a \\
\hline Angelalign & 1.89 & 0.31 & $\mathrm{~b}$ & 1.35 & 0.17 & $\mathrm{~b}$ & 1.29 & 0.28 & b & 0.47 & 0.14 & a \\
\hline Smartee & 1.61 & 0.30 & $\mathrm{~b}$ & 1.58 & 0.29 & $\mathrm{~b}$ & 1.01 & 0.20 & $b$ & 0.34 & 0.16 & a \\
\hline
\end{tabular}

IC, Intergroup comparison; SD, standard deviation.

* Intergroup comparison of colour differences regarding different aligner types according to the Tukey's multiple comparison test. Different letters indicate a statistically significant difference between aligner types in each solution at each time point $(P<0.05)$.
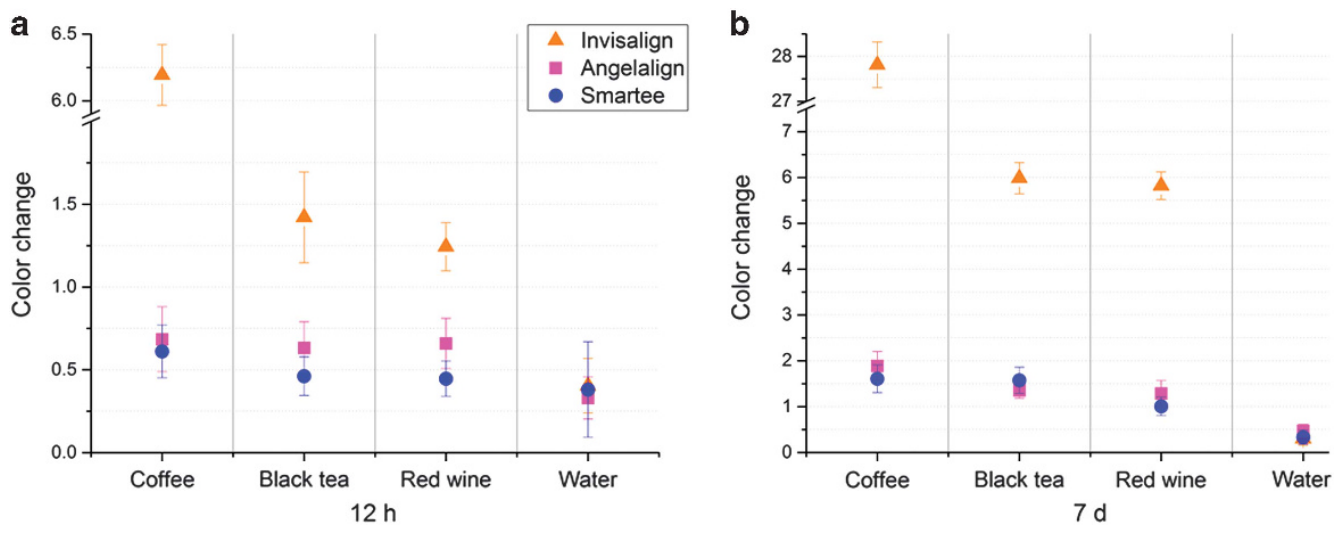

Figure 2 Colour changes in the three types of aligners stained in each solution. (a) Stained for $12 \mathrm{~h}$; (b) for $7 \mathrm{~d}$.

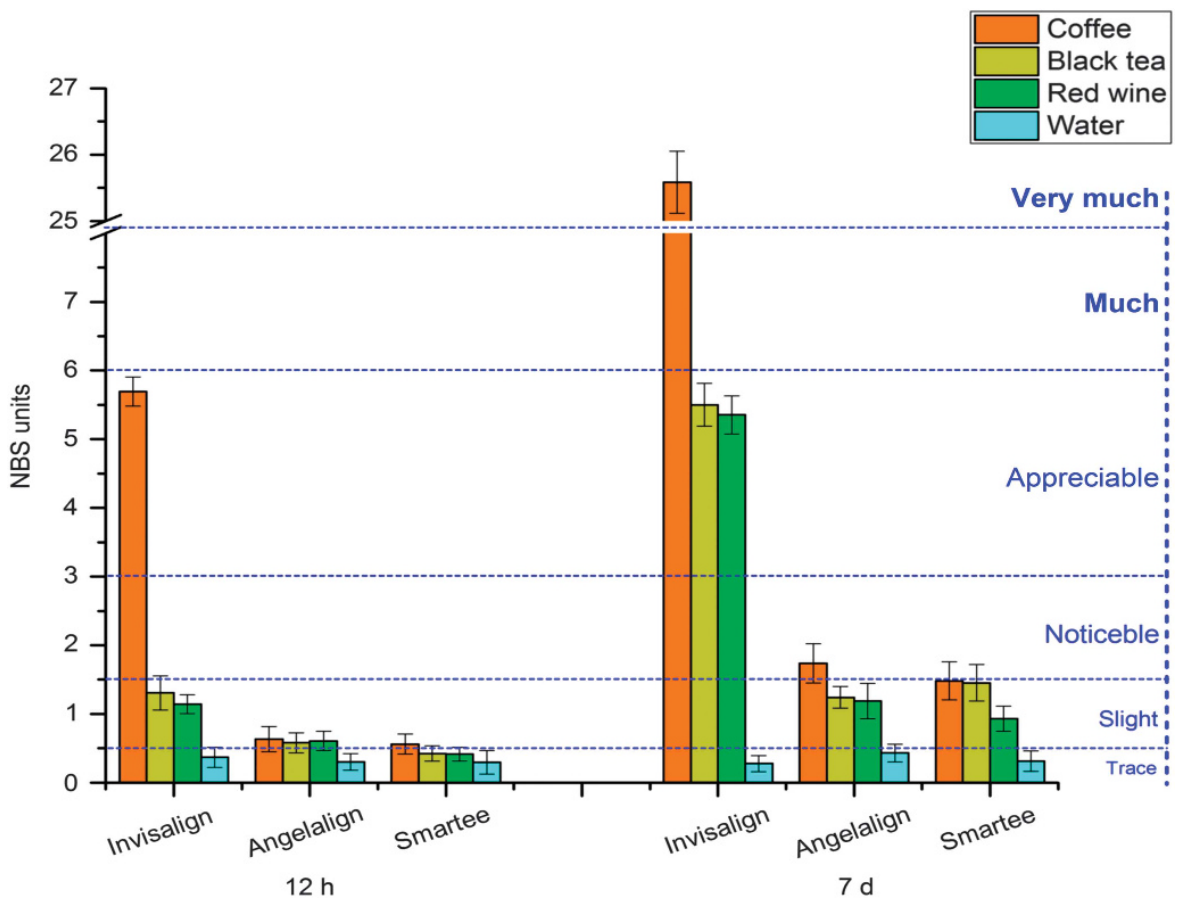

Figure 3 NBS units and colour change ratings of three types of aligners. NBS, National Bureau of Standards. 

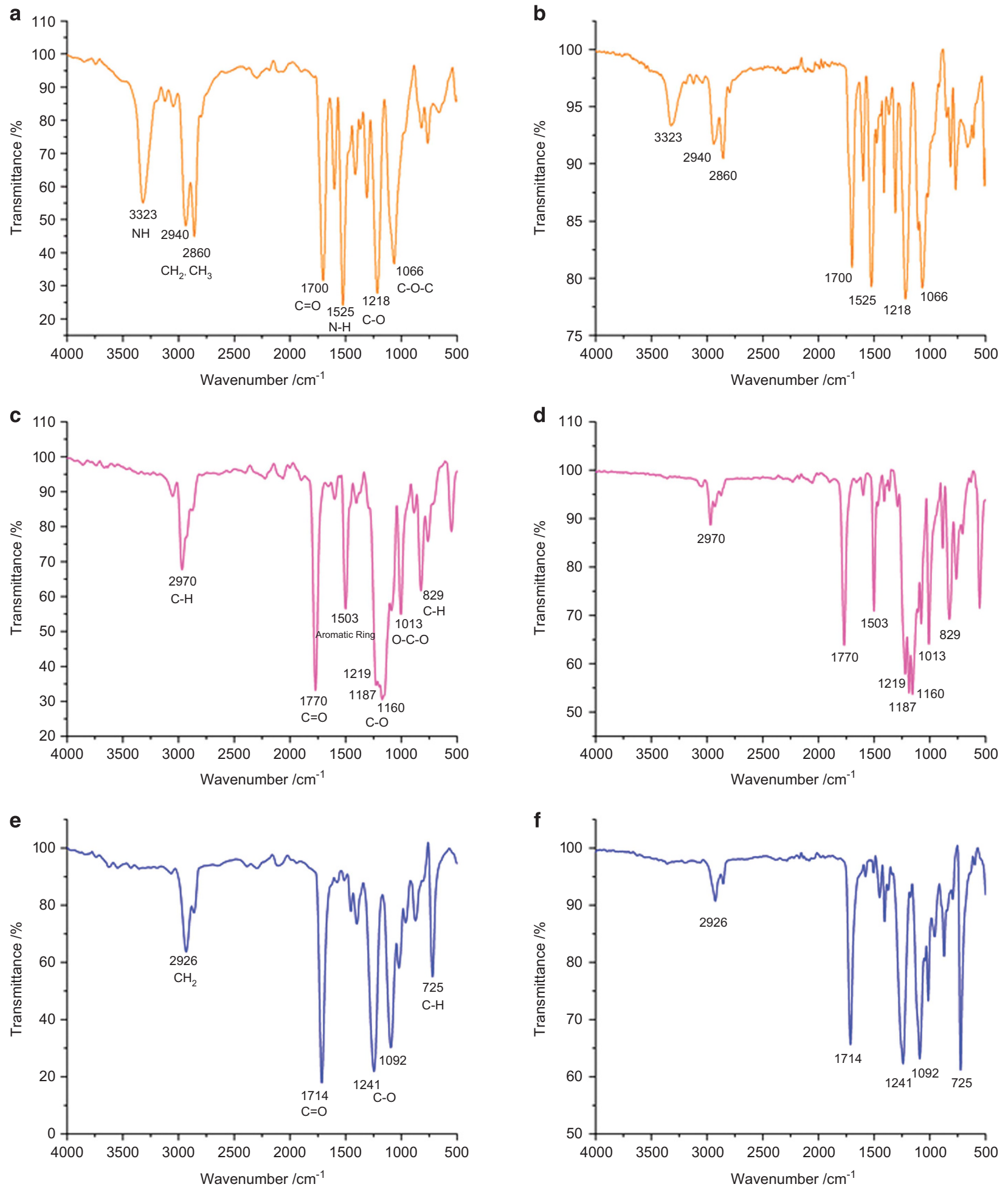

Figure 4 FT-IR spectra of the aligner materials. (a, b) Invisalign; (c, d) Angelalign; (e, f) Smartee. (a, c, e) Before staining; (b, d, f) after staining in coffee for $7 \mathrm{~d}$. FT-IR, Fourier transformation infrared.

shown in Figure 4. FT-IR analysis confirmed that the Invisalign aligners were a PU-based material, ${ }^{27-28}$ the Angelalign aligners and/or retainers were a polycarbonate (PC)-based material, and the Smartee aligners were a PETG-based material. There were no significant chemical differences in any of the three materials after the coffee staining process.
SEM examination

The SEM results revealed that the aligner materials exhibited rough surfaces after the 7-day coffee staining (Figure 5). The Invisalign material surface tended to be rougher and to exhibit more severe peeling and pore formation than the other two materials. The Smartee 

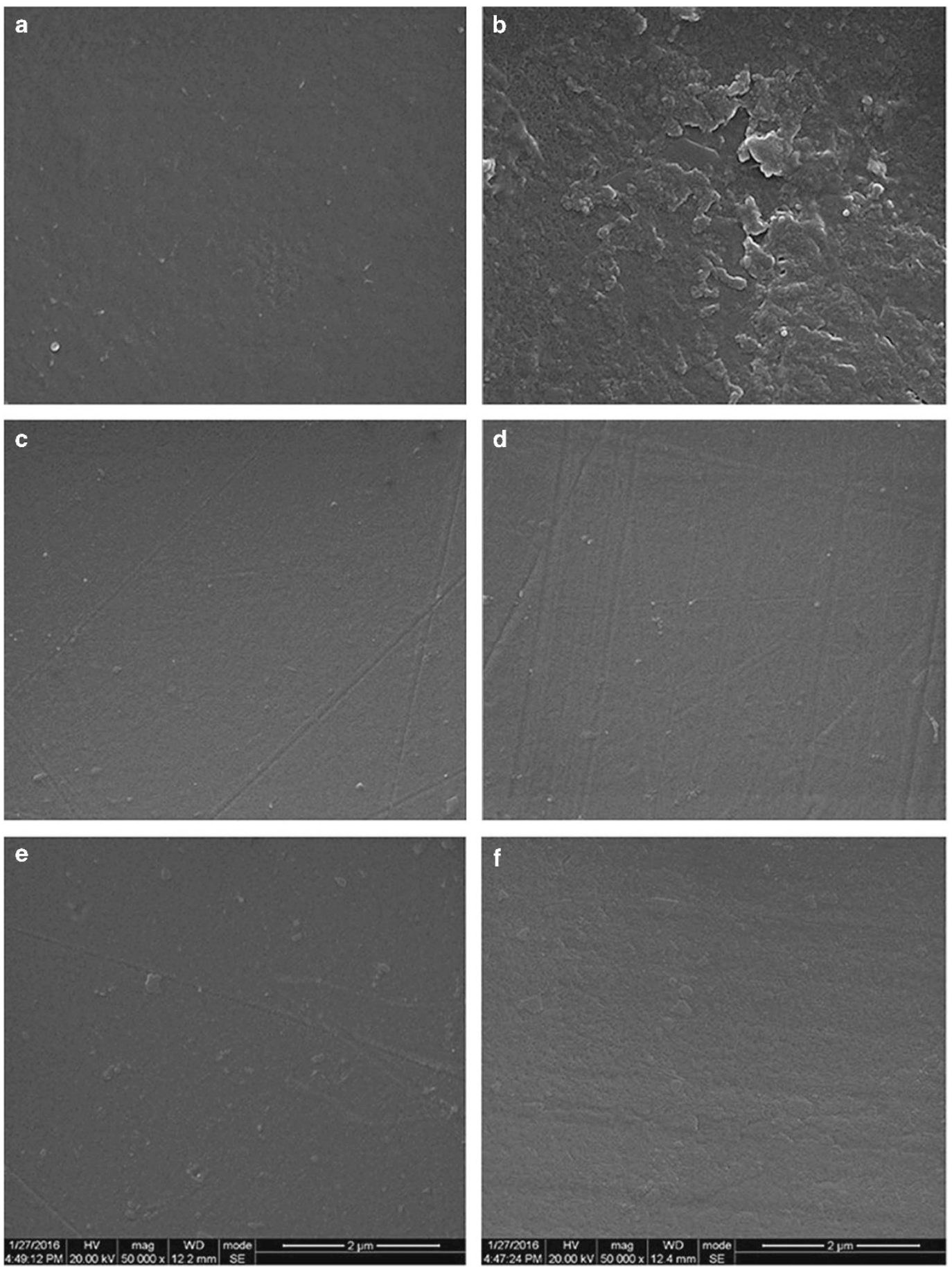

Figure 5 SEM examination of the surface alternations of the aligners staining in coffee for 7 d. (a, b) Invisalign; (c, d) Angelalign; (e, f) Smartee. (a, c, e) Before staining; (b, d, f) after staining. SEM, scanning electron microscopy.

material exhibited fewer or less pores and milder peeling, and the Angelalign material did not exhibit obvious pores or peeling.

\section{DISCUSSION}

PU, PC, and PETG are all amorphous polymers with a highly transparent appearance ${ }^{40-41}$ and the applications of such polymers can be found in almost all industrial branches and medical fields. ${ }^{13,42} \mathrm{PU}$ exhibits favourable features such as elasticity, flexibility, excellent mechanical properties, chemical resistance, and ease of processing. ${ }^{40,43}$ PC is widely used in orthodontic appliances, such as aesthetic brackets and clear aligners and retainers, owing to its good optical, physical, and chemical characteristics. ${ }^{4-46}$ PETG is a non-crystallising amorphous co-polymer of PET and is a relatively hard material with good mechanical properties, formability, fatigue resistance, and dimensional stability. ${ }^{13,17}$ Thus, all the three types of materials are widely used in the dental field. The FT-IR results suggested that all of the aligner materials were relatively chemically stable after staining in coffee for 7 days. ${ }^{31}$ 
The colour changes of the clear aligners may have been associated with pigment adsorption or penetration of the material surface when the aligners were immersed in the staining agents. This process might be related to the staining pigments in the agents and characteristics of the aligner materials. ${ }^{22-23,31,47-48}$ In a few previous studies, coffee has been identified as the strongest staining agent among common beverages. $^{31-32,49}$ The Invisalign instructions for patients suggest that drinking coffee with the aligner in the mouth should be avoided. ${ }^{50}$ The staining effect may be due to pigments, such as yellow dye in coffee and tea solutions. ${ }^{48}$ The present study revealed that all of the tested aligners exhibited almost no colour change in the distilled water, and the PU-, PC-, and PETG-based aligners exhibited high colour stabilities after 7 days of immersion in a colourless solution. However, after exposure to staining solutions, such as coffee, black tea, and red wine, the aligners exhibited colour changes, although some of these changes were very slight.

The differences in the colour changes of the three types of aligners may have been due to their different polymer-based materials, which might have different susceptibilities to staining agents. The PU-based Invisalign aligners exhibited significantly greater colour change values than those of the PC and PETG-based aligners. Similarly, previous studies have found that $\mathrm{PU}$ is vulnerable to pigment adsorption and generally does not provide adequate colour stability. ${ }^{22-23,51}$ PU-based orthodontic retainers and ligatures exhibit visible colour changes after immersion in beverages. ${ }^{23,31}$ In contrast, PC-based aesthetic wires have been reported to maintain high levels of colour stability. ${ }^{19} \mathrm{PC}$ is generally recognised as a low-adsorption material ${ }^{43}$ that can provide good colour stability. One study has also indicated that PU materials exhibit greater water absorption rates than PC and PETG in a simulated intraoral environment. Water absorption results from the attachment of the water molecules to the material surface and subsequent absorption or penetration into the material, ${ }^{52}$ which might facilitate the adsorption and attachment of the pigments in the solutions to the material surfaces. Moreover, PU polymers contain the surface polar group '-NHCOO-', and this structural unit is prone to the formation of hydrogen bonds that interact with hydrophilic pigments in solutions, ${ }^{53}$ thus facilitating pigment adsorption onto the material. In contrast, PC and PETG polymers contain surface groups, such as '-COO-' and 'C-O-C', that are less polar than '-NHCOO-'.53

Furthermore, the variations in the colour changes among the three types of aligners may be explained by the materials' surface characteristics, such as roughness, which might also accelerate pigment accumulation. ${ }^{23}$ The SEM results in the present study suggested that the PU-based material surface tends to be rougher with more severe peeling. The porosities of the aligner surfaces may be the result of the degradation of the materials. ${ }^{22}$ When aligners are worn in the oral cavity, their surface roughness would be more prominent because of microcracks and abrasions from speech, swallowing, and bruxism, which could enhance pigmentation and lead to the loss of the transparency of the thermoplastic aligner. ${ }^{23,54}$

The present results suggested that all the three types of aligners exhibit minimal colour changes after 12-h staining, with the exception of the Invisalign aligners stained by coffee. It has been reported that $50 \%$ of Americans adults drink coffee every day and consume an average of 3.2 cups per day, drinking each cup over the course of 15 min. ${ }^{25,32}$ According to a previous calculation, ${ }^{32} 12 \mathrm{~h}$ of in vitro staining simulates 2 weeks' coffee exposure time in clinical aligner usage. This result illustrates the aesthetic stabilities of the three types of clear aligners, particularly the PC and PETG-based aligners. However, to maintain the colour stabilities of the aligners for clinical aesthetics and to protect the mechanical properties of aligners, patients should follow the instructions to remove the aligners before eating and drinking. Orthodontists are supposed to inform patients of the instructions, especially patients who are coffee drinkers.

Orthodontic aligner appearance may influence social perceptions and thus is a factor in the selection of aligners by patients. ${ }^{1}$ In contrast, the functional performance of clear aligners in terms of mechanical properties and force delivery features are also vital for orthodontic treatment. Although the colour of the PU-based aligners is relatively less stable, particularly after coffee exposure, the soft PU elastomer aids in improving the elasticity and is able to provide continuous light force to the teeth, which is beneficial for orthodontic tooth movement. ${ }^{14,30,40}$ Considering the aesthetic and functional improvements of aligner materials, further studies should be conducted to improve the colour stability and mechanical properties of aligner material. Because the pigment adsorption is related to polymer surface polar groups and roughness, increases in the surface hydrophobicity and integrity might reduce pigment adsorption and penetration. Studies have been conducted regarding the modification of material surfaces with low surface energy by using fluorinated or silicon compounds to fabricate superhydrophobic surfaces. ${ }^{55-56}$ Thus, fluorinated or silicon compound-modified PU might have the potential to achieve better colour stability. ${ }^{57-58}$ Researchers have also attempted to improve the functional properties of the relatively colour-stable PETG material. Using the blending modification method, PETG/PC/thermoplastic PU polymer has been produced and used as an aligner material with optimal mechanical properties. ${ }^{17}$

\section{CONCLUSIONS}

As aesthetic orthodontic appliances, the three types of clear aligners (Invisalign, Angelalign and Smartee) exhibited colour stability after 12-h immersions, with the exception of the Invisalign aligners stained with coffee. The Invisalign aligners were more prone to pigmentation than the Angelalign and Smartee aligners. Aligner materials may be improved in terms of their aesthetic stability properties.

\section{ACKNOWLEDGEMENTS}

We acknowledge the significant contributions of Mr. Tao Zhang, Department of Statistics, West China School of Public Health, Sichuan University and Professor Hang Wang from the Department of Prosthodontics, West China School of Stomatology, Sichuan University. We also thank Professor Hong Tan from the College of Polymer Science and Engineering, State Key Laboratory of Polymer Materials Engineering, Sichuan University. This work was funded by the Orthodontic National Key Clinical Specialty Construction Program of China, the West China Hospital of Stomatology Sichuan University and was also supported by grants from the National Natural Science Foundation of China (Grant No. 31470904 and 81470722).

1 Meade MJ, Millett DT, Cronin M. Social perceptions of orthodontic retainer wear. Eur J Orthod 2014; 36(6): 649-656.

2 Cooper-Kazaz R, Ivgi I, Canetti L et al. The impact of personality on adult patients' adjustability to orthodontic appliances. Angle Orthod 2013; 83(1): 76-82.

3 Maspero C, Farronato D, Giannini L et al. Orthodontic treatment in elderly patients. Prog Orthod 2010; 11(1): 62-75.

4 Ghafari JG. Centennial inventory: the changing face of orthodontics. Am J Orthod Dentofacial Orthop 2015; 148(5): 732-739.

5 Malik $\mathrm{OH}$, McMullin A, Waring DT. Invisible orthodontics part 1: invisalign. Dent Update 2013; 40(3). 203-204, 207-210, 213-215.

6 Rosvall MD, Fields HW, Ziuchkovski J et al. Attractiveness, acceptability, and value of orthodontic appliances. Am J Orthod Dentofacial Orthop 2009; 135(3): $276 . \mathrm{e} 1$ -276.e12, discussion 276-277.

7 Shalish M, Cooper-Kazaz R, Ivgi I et al. Adult patients' adjustability to orthodontic appliances. Part I: a comparison between Labial, Lingual, and Invisalign ${ }^{\mathrm{TM}}$. Eur J Orthod 2012; 34(6): 724-730. 
8 Martorelli M, Gerbino S, Giudice M et al. A comparison between customized clear and removable orthodontic appliances manufactured using RP and CNC techniques. Dent Mater 2013; 29(2): e1-e10.

9 Pithon MM. A modified thermoplastic retainer. Prog Orthod 2012; 13(2): 195-199.

10 Ercoli F, Tepedino M, Parziale V et al. A comparative study of two different clear aligner systems. Prog Orthod 2014; 15(1): 31

11 Kravitz ND, Kusnoto B, BeGole E et al. How well does Invisalign work? A prospective clinical study evaluating the efficacy of tooth movement with Invisalign. Am J Orthod Dentofacial Orthop 2009; 135(1): 27-35.

12 Hahn W, Engelke B, Jung $\mathrm{K}$ et al. Initial forces and moments delivered by removable thermoplastic appliances during rotation of an upper central incisor. Angle Orthod 2010; 80(2): 239-246.

13 Fujiwara K, Fukuhara T, Niimi K et al. Mechanical evaluation of newly developed mouthpiece using polyethylene terephthalate glycol for transoral robotic surgery. J Robot Surg 2015; 9(4): 347-354.

14 Clements KM, Bollen AM, Huang G et al. Activation time and material stiffness of sequential removable orthodontic appliances. Part 2: dental improvements. $A m \mathrm{~J}$ Orthod Dentofacial Orthop 2003; 124(5): 502-508.

$15 \mathrm{Ahn} \mathrm{HW}$, Ha HR, Lim HN et al. Effects of aging procedures on the molecular, biochemical, morphological, and mechanical properties of vacuum-formed retainers. J Mech Behav Biomed Mater 2015; 51: 356-366.

16 Drake CT, McGorray SP, Dolce $\mathrm{C}$ et al. Orthodontic tooth movement with clear aligners ISRN Dent 2012; 2012: 657973.

17 Zhang N, Bai $Y$, Ding $X$ et al. Preparation and characterization of thermoplastic materials for invisible orthodontics. Dent Mater J 2011; 30(6): 954-959.

18 Ziuchkovski JP, Fields HW, Johnston WM et al. Assessment of perceived orthodontic appliance attractiveness. Am J Orthod Dentofacial Orthop 2008; 133(4 Suppl): S68-S78.

19 Inami T, Tanimoto $\mathrm{Y}$, Minami N et al. Color stability of laboratory glass-fiber-reinforced plastics for esthetic orthodontic wires. Korean J Orthod 2015; 45(3): 130-135.

20 Dietschi D, Campanile G, Holz J et al. Comparison of the color stability of ten newgeneration composites: an in vitro study. Dent Mater 1994; 10(6): 353-362.

21 Ardeshna AP, Vaidyanathan TK. Colour changes of orthodontic elastomeric module materials exposed to in vitro dietary media. J Orthod 2009; 36(3): 177-185.

$22 \mathrm{Kim}$ SH, Lee YK. Measurement of discolouration of orthodontic elastomeric modules with a digital camera. Eur J Orthod 2009; 31(5): 556-562.

23 Fernandes AB, Ruellas AC, Araújo MV et al. Assessment of exogenous pigmentation in colourless elastic ligatures. J Orthod 2014; 41(2): 147-151.

24 Tsomos G, Ludwig B, Grossen J et al. Objective assessment of patient compliance with removable orthodontic appliances: a cross-sectional cohort study. Angle Orthod 2014; 84(1): 56-61.

25 The National Coffee Association and The Specialty Coffee Association of America Coffee Statistics. Available at http://www.e-importz.com/coffee-statistics.php (accessed on 15 July 2015).

26 Wriedt S, Schepke U, Wehrbein $\mathrm{H}$. The discoloring effects of food on the color stability of esthetic brackets-an in-vitro study. J Orofac Orthop 2007; 68(4): 308-320.

27 Gerard BT, Teske L, Eliades G et al. Do the mechanical and chemical properties of Invisalign appliances change after use? A retrieval analysis. Eur J Orthod 2016; 38(1): 27-31.

28 Alexandropoulos A, Al Jabbari YS, Zinelis S et al. Chemical and mechanical characteristics of contemporary thermoplastic orthodontic materials. Aust Orthod $\mathrm{J}$ 2015; 31(2): 165-170.

29 Premaraj T, Simet S, Beatty $M$ et al. Oral epithelial cell reaction after exposure to Invisalign plastic material. Am J Orthod Dentofacial Orthop 2014; 145(1): 64-71.

30 Elkholy F, Panchaphongsaphak T, Kilic F et al. Forces and moments delivered by PET-G aligners to an upper central incisor for labial and palatal translation. J Orofac Orthop 2015; 76(6): 460-475

31 Zafeiriadis AA, Karamouzos A, Athanasiou AE et al. In vitro spectrophotometric evaluation of Vivera clear thermoplastic retainer discolouration. Aust Orthod J 2014, 30(2): 192-200

32 de Oliveira CB, Maia LG, Santos-Pinto A et al. In vitro study of color stability of polycrystalline and monocrystalline ceramic brackets. Dental Press J Orthod 2014; 19 (4): 114-121.

33 Johnston WM. Color measurement in dentistry. J Dent 2009; 37(Suppl 1): e2-e6.

34 Cörekçi B, Irgın C, Malkoç S et al. Effects of staining solutions on the discoloration of orthodontic adhesives: an in-vitro study. Am J Orthod Dentofacial Orthop 2010; 138(6): 741-746.

35 Nimeroff I. Colorimetry. Natl Bureau Stand Monogr 1968; 47: 104.

36 Koksal T, Dikbas I. Color stability of different denture teeth materials against various staining agents. Dent Mater J 2008; 27(1): 139-144.
37 da Silva DL, Mattos CT, de Araújo MV et al. Color stability and fluorescence of different orthodontic esthetic archwires. Angle Orthod 2013; 83(1): 127-132.

38 Oh SY, Kang MS, Knowles JC et al. Synthesis of bio-based thermoplastic polyurethane elastomers containing isosorbide and polycarbonate diol and their biocompatible properties. J Biomater App/ 2015; 30(3): 327-337.

39 Kosobrodova E, Jones RT, Kondyurin A et al. Orientation and conformation of anti-CD34 antibody immobilised on untreated and plasma treated polycarbonate. Acta Biomater 2015; 19: 128-137.

40 Špírková M, Pavličević J, Strachota A et al. Novel polycarbonate-based polyurethane elastomers: composition-property relationship. Eur Polym J 2011; 47(5): 959-972.

41 Ahn HW, Kim KA, Kim SH. A new type of clear orthodontic retainer incorporating multilayer hybrid materials. Korean J Orthod 2015; 45(5): 268-272.

42 Matsui S, Umezaki E, Komazawa $D$ et al. Evaluation of mechanical properties of esthetic brackets. J Dent Biomech 2015; 6: 1758736015574401.

43 Selvakumar M, Jaganathan SK, Nando GB et al. Synthesis and characterization of nove polycarbonate based polyurethane/polymer wrapped hydroxyapatite nanocomposites: mechanical properties, osteoconductivity and biocompatibility. J Biomed Nanotechnol 2015; 11(2): 291-305.

44 Kloukos D, Taoufik E, Eliades $T$ et al. Cytotoxic effects of polycarbonate-based orthodontic brackets by activation of mitochondrial apoptotic mechanisms. Dent Mater 2013; 29(3): e35-e44

45 Hamanaka I, Iwamoto M, Lassila L et al. Influence of water sorption on mechanical properties of injection-molded thermoplastic denture base resins. Acta Odontol Scand 2014; 72(8): 859-865.

46 Lombardo L, Martines E, Mazzanti V et al. Stress relaxation properties of four orthodontic aligner materials: a 24-hour in vitro study. Angle Orthod 2016; doi:10.2319/113015-813.1.

47 Erdemir U, Yildiz E, Eren MM. Effects of sports drinks on color stability of nanofilled and microhybrid composites after long-term immersion. J Dent 2012; 40(Suppl 2): e55-e63.

$48 \mathrm{Um}$ CM, Ruyter IE. Staining of resin-based veneering materials with coffee and tea. Quintessence Int 1991; 22(5): 377-386.

49 Fernandes AB, Ribeiro AA, Araujo MV et al. Influence of exogenous pigmentation on the optical properties of orthodontic elastic ligatures. J Appl Oral Sci 2012; 20(4): $462-466$.

50 Anon. Questions and Answers for Invisalign Treament. Available at http://www. invisalign. com.cn/faq (accessed 15 July 2015).

51 Oliveira AS, Kaizer MR, Salgado VE et al. Influence of whitening and regular dentifrices on orthodontic clear ligature color stability. J Esthet Restor Dent 2015; 27(Suppl 1): S58-S64.

52 Ryokawa H, Miyazaki Y, Fujishima A et al. The mechanical properties of denta thermoplastic materials in a simulated intraoral environment. Orthod Waves 2006; 65 (2): 64-72.

53 Gunter O. Polyurethane Handbook. Munich: Hanser Publishers, 1994.

54 Schuster S, Eliades G, Zinelis S et al. Structural conformation and leaching from in vitro aged and retrieved Invisalign appliances. Am J Orthod Dentofacial Orthop 2004; 126 (6): 725-728.

55 Han JT, Zheng Y, Cho JH et al. Stable superhydrophobic organic-inorganic hybrid films by electrostatic self-assembly. J Phys Chem B 2005; 109(44): 20773-20778.

56 Sun $T$, Wang G, Liu $\mathrm{H}$ et al. Control over the wettability of an aligned carbon nanotube film. J Am Chem Soc 2003; 125(49): 14996-14997.

$57 \mathrm{Tan} \mathrm{H}$, Xie X, Li J et al. Synthesis and surface mobility of segmented polyurethanes with fluorinated side chains attached to hard blocks. Polymers 2004; 45(5): $1495-1502$.

58 Yang J, Gao Y, Li J. Synthesis and microphase separated structures of polydimethylsiloxane/polycarbonate-based polyurethanes. RSC Adv 2013; 3(22): 8291-8297.

(c) $($ i) $\Theta$ This work is licensed under a Creative Commons AttributionBY NC ND NonCommercial-NoDerivs 4.0 International License. The images or other third party material in this article are included in the article's Creative Commons license, unless indicated otherwise in the credit line; if the material is not included under the Creative Commons license, users will need to obtain permission from the license holder to reproduce the material. To view a copy of this license, visit http:// creativecommons.org/licenses/by-nc-nd/4.0/

(C) The Author(s) 2016 